\title{
Angiosarcome de grade intermédiaire de malignité : un cas
}

\author{
Landric C, El Okeily M, Fricain JC. \\ (UFR d'Odontologie, Bordeaux)
}

Les sarcomes sont des tumeurs malignes dont l'incidence est de 5000 cas par an (Sim 1994). L'angiosarcome ou hémangioendothéliome est une tumeur rare de l'endothélium vasculaire observée le plus souvent sur la peau et sur le cuir chevelu. Les cas décrits dans la littérature soulignent le pronostic sombre et l'issue rapidement mortelle.

Le cas que nous rapportons présente un intérêt de par sa rareté de survenue dans la cavité buccale, mais également de part l'aspect bénin de la lésion, ce qui a retardé le diagnostic.

Un patient de 90 ans avec antécédents médicaux d'hypertension artérielle, hyperuricémie, insuffisance cardiaque, traité par FUROSEMIDE ${ }^{\circledR}$, ALLOPURINOL $^{\circledR}$, PREVISCAN $^{\circledR}$, a consulté pour un nodule palatin violacé, lisse de $3 \mathrm{~cm}$ de diamètre. La lésion était circonscrite et bien limitée. La palpation des aires ganglionnaires cervico faciales était libre. Le diagnostic clinique initial a été orienté vers un kyste mucoïde du voile du palais. Le caractère hématique de la lésion avait été attribué au traitement par anti vitamine K. L'absence de gêne fonctionnelle n'a pas indiqué de traitement chirurgical de la lésion. Une simple surveillance a été décidée. 3 mois plus tard, une adénopathie sous angulo mandibulaire droite controlatérale est apparue. Elle a fait suspecter une lésion maligne. L'IRM avec produit de contraste a mis en évidence une lésion palatine infiltrante. Le patient a été opéré sous anesthésie générale pour l'exérèse de la lésion palatine associée à une exérèse ganglionnaire. L'examen anatomopathologique de la lésion palatine a mis en évidence une production tumorale polipoïde de forme fuso-cellulaire, largement ulcérée en surface avec de nombreux remaniements inflammatoires et une extravasation d'hématies. De nombreuses atypies cytonucléaires accompagnées de nombreuses mitoses étaient observées. L'étude immunohistochimique a révélé que les marqueurs vasculaires (CD 31 et CD 34) étaient positifs au niveau des cellules tumorales ce qui a permis de confirmer le diagnostic d'angiosarcome. Le KI 67 a montré un index de prolifération élevé, de l'ordre de $30 \%$. Ces éléments ont permis le diagnostic d'angiosarcome de grade intermédiaire de malignité. L'analyse anatomopathologique du ganglion a révélé une métastase de l'angiosarcome. L'angiosarcome est de mauvais pronostic mais compte tenu de l'âge du patient et de l'absence d'autres éléments tumoral flagrants, aucun traitement complémentaire n'a été proposé.

Une surveillance avec un contrôle clinique tous les 3 mois et radio pulmonaire tous les 6 mois a été instauré.

Les angiosarcomes représentent 0,5 à $1 \%$ des tumeurs malignes de l'adulte et $15 \%$ des tumeurs malignes de l'enfant. Cette tumeur se développe principalement sur un lymphœdème chronique post-radique et chez le sujet âgé en région céphalique. II est aussi associée à une exposition au chlorure de vinyle, à l'arsenic et au dioxyde de thorium (Agency for Toxic Substances, 2006) La plupart des angiosarcomes sont des tumeurs de haut grade qui sont agressifs et à croissance rapide. Dans la cavité buccale, l'angiosarcome est de forme variée: nodulaire, de couleur rouge, bleu ou violet (Abdullah BH 2000). Les aspects cliniques étant très hétérogènes, la biopsie est l'examen discriminant de cette tumeur qui doit être évoquée chez les patients âgés en l'absence de guérison d'une lésion d'une lésion nodulaire violacée. 